\title{
Clinical management and genetic architecture of severe hypercholesterolemia in Finland
}

Nella Junna ${ }^{1}$, Pietari Ripatti ${ }^{1}$, Ida Surakka ${ }^{1,2}$, Samuli Ripatti ${ }^{1,3}$, Elisabeth Widén ${ }^{1}$

\section{Aim}

To characterize the prevalence, clinical management, and underlying genetic architecture of severe hypercholesterolemia, we studied individuals with $\mathrm{LDL}$ cholesterol $\geq 5 \mathrm{mmol} / \mathrm{l}$ in the prospective GeneRISK study.

\section{Study population}

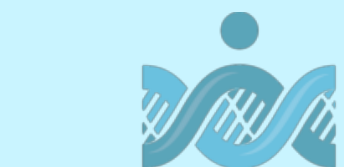

\section{GeneRISK}

The GeneRISK study

- Randomly ascertained participants from Southern Finland

- 7349 subjects, aged $45-65$ years, $36,1 \%$ males

- Clinical checkup, blood lipids, questionnaire at baseline, follow-up after 1,5 years

\section{Hypercholesterolemia and 10-year risk for} cardiovascular diseases (CVD)

\section{CVD risk among cases with}

LDL-C $\geq 5 \mathrm{mmol} / \mathrm{l}(\mathrm{N}=366)$

$11,1 \%$ of subjects at high CVD risk

(risk $>10 \%$ ) had LDL-C $\geq 5 \mathrm{mmol} / \mathrm{l}$

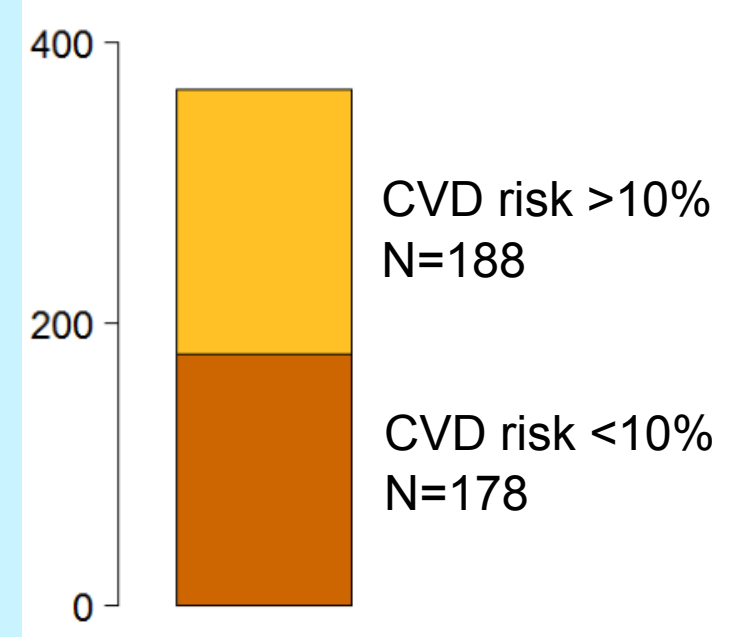

LDL-C $\geq 5 \mathrm{mmol} / \mathrm{l}$ $5,0 \%$ of the population

\section{Prevalence of severe}

hypercholesterolemia:

- Mean LDL-C: 3,4 mmol//

$-5,0 \%(N=366)$ with

$\mathrm{LDL}-\mathrm{C} \geq 5 \mathrm{mmol} / /$
All GeneRISK participants received information of their personal 10-year CVD risk, which is based on age, smoking, blood pressure, lipids and a polygenic risk score (49 000 SNPs) (3).

\section{Hypercholesterolemia mutations}

\section{Mutation screening:}

Genotyping and whole-exome sequencing $(\mathrm{N}=206, \mathrm{LDL}-\mathrm{C} \geq 5 \mathrm{mmol} / \mathrm{l})$

Results:

$>$ No carriers of the 7 Finnish

$L D L R$ founder mutations

$>$ A novel, likely pathogenic mutation LDLR R574L identified:

- Once previously associated with familial hypercholesterolemia (4)

- Frequencies in gnomAD database: Finnish: 0,0002

other populations: 0

- Pathogenic in silico predictions in all 8 tested programs
Management of hypercholesterolemia

At baseline

CVD risk $>10 \%$, CVD risk $<10 \%$,

LDL $\geq 5 \quad$ LDL $\geq 5$

N (male \%)

$188(45,2 \%) \quad 178(33,1 \%)$

Age (years)

$57,6 \pm 5,1 \quad 55,3 \pm 5,2^{* * *}$

BMI $\left(\mathbf{k g} / \mathrm{m}^{2}\right)$

$28,3 \pm 4,1$

$27,5+4,5^{*}$

Systolic BP $(\mathrm{mmHg})$

$134,8 \pm 16,7 \quad 127,7 \pm 17,0$ ***

Diastolic BP $(\mathrm{mmHg})$

$89,7 \pm 10,3 \quad 85,1 \pm 10,3^{* * *}$

Total cholesterol (mmol/l)

HDL-C (mmol/l)

$8,1 \pm 0,9 \quad 7,4 \pm 0,3^{* * *}$

LDL-C (mmol/l)

$1,6 \pm 0,5$

$1,5 \pm 0,3$

$5,8 \pm 0,8$

$5,3+0,2^{* * *}$

Triglycerides ( $\mathrm{mmol} / \mathrm{l})$

$1,7 \pm 0,6$

$1,4 \pm 0,5^{\star \star *}$

Smoking (yes)

$25,0 \%$

$14,0 \% *$

BP lowering

medication (yes)

Lipid lowering

medication (yes)

Previous

$20,7 \%$

$13,5 \%$

$4,3 \%$

$3,9 \%$

hypercholesterolemia (yes)

$86,7 \%$

$84,3 \%$

\section{At follow-up}

N (male \%)

My results were surprising

My results were concerning

My results were useful

Seen a doctor during follow-up

Lipid lowering medication

Changes in:

BMI $\left(\mathrm{kg} / \mathrm{m}^{2}\right)$

Total cholesterol (mmol/l)

HDL-C (mmol/I)

LDL-C (mmol/l)

Triglycerides (mmol/l)

${ }^{*} p<0,05 ;{ }^{* *} p<0,01 ;{ }^{* * *} p<0,001$
CVD risk $>10 \%$, CVD risk $<10 \%$, LDL $\geq 5$

$\mathrm{LDL} \geq 5$

$127(44,9 \%)$

$114(34,3 \%)$

$48,8 \%$

$21,9 \%^{* \star \star}$

$58,3 \%$

$35,1 \%$ **

$88,2 \%$

$90,4 \%$

$37,8 \%$

$14,9 \%^{* * *}$

$33,9 \%$

$17,9 \%$ *

$0 \pm 1,4$

$0,2 \pm 1,3^{*}$

$-1,6 \pm 1,6$

$-0,8 \pm 1,1^{* * *}$

$0 \pm 0,3$

$0 \pm 0,2$

$-1,6 \pm 1,5$

$-0,8 \pm 1,1^{* * *}$

$0 \pm 0,6$

$0,1 \pm 0,5^{*}$

\section{Conclusions}

- Severe hypercholesterolemia is poorly treated in Southern Finland - A significant proportion of patients are unaware of their CVD risk

- The contribution of known hypercholesterolemia-associated mutations in Southern Finland appears smaller than previously anticipated

- There is an unmet need to further characterize causal variants and to raise awareness among caretakers to adequately treat patients. 